\title{
A novel round-robin based multicast scheduling algorithm for 100 Gigabit Ethernet switches
}

\author{
Yu, Hao; Ruepp, Sarah Renée; Berger, Michael Stübert
}

Published in:

proceedings INFOCOM

Link to article, DOI:

10.1109/INFCOMW.2010.5466651

Publication date:

2010

Document Version

Publisher's PDF, also known as Version of record

Link back to DTU Orbit

Citation (APA):

Yu, H., Ruepp, S. R., \& Berger, M. S. (2010). A novel round-robin based multicast scheduling algorithm for 100 Gigabit Ethernet switches. In proceedings INFOCOM (pp. 1-2). IEEE.

https://doi.org/10.1109/INFCOMW.2010.5466651

\section{General rights}

Copyright and moral rights for the publications made accessible in the public portal are retained by the authors and/or other copyright owners and it is a condition of accessing publications that users recognise and abide by the legal requirements associated with these rights.

- Users may download and print one copy of any publication from the public portal for the purpose of private study or research.

- You may not further distribute the material or use it for any profit-making activity or commercial gain

- You may freely distribute the URL identifying the publication in the public portal

If you believe that this document breaches copyright please contact us providing details, and we will remove access to the work immediately and investigate your claim. 


\title{
A Novel Round-Robin Based Multicast Scheduling Algorithm for 100 Gigabit Ethernet Switches
}

\author{
$\mathrm{Hao} \mathrm{Yu}$ \\ Supervisors: Sarah Ruepp, Michael S. Berger \\ Technical University of Denmark \\ 2800 Kgs. Lyngby, Denmark \\ haoyu@fotonik.dtu.dk
}

\begin{abstract}
This paper proposes a round-robin based multicast scheduling algorithm for high-speed input-queued switches. Fanout information of each head-of-line cell is examined by the packet scheduler to form a matrix called Traffic Matrix. A subscheduler for each column executes the round-robin scheduling algorithm and scheduling decisions are collected into the Decision Matrix. To avoid unnecessary multiple transmissions of a multicast cell, the sync mechanism is introduced after the Decision Matrix is formed. By simulation, the results demonstrate that the number of transmissions is effectively decreased by sync while maintaining the same output utilization.
\end{abstract} switch

Keywords: multicast scheduling; round-robin; high-speed

\section{INTRODUCTION}

Multicast capability of a switch is of crucial importance to the communication networks in the foreseeable future. As broadband services such as video conferencing, online gaming and IPTV becoming popular, it is no longer a resource-efficient way to use unicast for these services. Multicast, on the other hand, is able to reduce the network traffic load and multicast latency.

Intensive research has been carried out in the area of scheduling for unicast multistage switches. However, for multicast switch scheduling, research in this area is still at the primary stage. Based on the position of the buffer which is used to store blocked packets, switches are mainly categorized as input-queued (IQ), output-queued (OQ), and virtual outputqueued (VOQ). OQ is least favored because of the poor scalability that output memories are required to run with a speedup of $N$, where $N$ denotes the number of input ports. On the contrary, IQ is advantageous because no speedup is required for the memory access. However, IQ suffers from head-of-line (HOL) blocking problem that reduces the throughput to $58.6 \%$ [1]. By creating a queue for each output within each input queue, VOQ is able to solve the HOL problem. Using VOQ for multicast traffic, however, requires $2^{N}-1$ queues in each input for all the possible combinations of destinations, which greatly reduces the scalability. Pan et al [2], [3] propose a way to reduce the number of required queues to $N$ by introducing the concept of address cells and data cells. However, the risk of multiple transmissions of a multicast packet is not eliminated. In this paper, we propose a multicast scheduling algorithm for IQ $M \times N$ switches based on the roundrobin discipline.

\section{ROUND-ROBIN BASED MULTICAST SCHEDULING AGLORITHM}

Normally, a packet is fragmented into fixed-size cells at the input before traversing the switch fabric and then defragmented at the output. The proposed multicast scheduling algorithm is applied after the fragmentation, and is based on the assumption that multicast cells in an input queue are stored in a first-infirst-out (FIFO) fashion. A multicast cell carries a fan-out vector which records the output ports the cell should be sent to. We assume that the fan-out vector has $N$ bits as $b_{N-1} b_{N-2} \ldots b_{l} b_{0}$, each of which represents an output port. With a bit set to 1 , the multicast cell should be sent to the corresponding output port. A broadcast cell will then have a fan-out vector of all $1 \mathrm{~s}$. Before a time slot of cell transmission begins, the packet scheduler examines the fan-out information of the HOL cell in each input in parallel. Information is then used to form a matrix, called Traffic Matrix (TM) with $M$ rows and $N$ columns, and totally $M \cdot N$ elements, $E_{i, j}(0 \leq i \leq N-1,0 \leq j \leq M$ 1). Take a $4 \times 4$ switch for instance, if the fan-out vectors of Input 0 to 3 are $1110,1100,0101$, and 0011 respectively, then the $T M$ will be constructed as shown in Fig. 1.

Each column has a round-robin subscheduler to make independent scheduling decisions. After the decisions are made, a Decision Matrix (DM) is then formed, containing the scheduling results as shown in Fig. 1. Based on this matrix, the scheduler reads each row and sends the corresponding cell from each input. If the row equals to the fan-out of the cell, the cell will be removed from the HOL position and sent to its destination ports. If the row is a subset of the fan-out vector, the cell will only be sent to the granted destinations and remains in its position with an updated fan-out vector. An allzero row indicates no cell should be released from the input.

Since the subschedulers are independent and execute algorithms in parallel, unnecessary multiple transmissions of a multicast cell may occur. To eliminate this, the scheduler rearranges the $D M$ before releasing any cell. This process is defined as sync. The role of arbiter is passed to each subscheduler in a round-robin fashion. Once a subscheduler becomes the arbiter, the scheduler will use the row that the arbiter's decision points to as a base and compare others'

This work was supported in part by the Danish National Advanced Technology Foundation in the project the Road to 100 Gigabit Ethernet 
decision with it. If the scheduling decision of a nonarbiter is contained in the base, the decision will be ignored and integrated to the base. As shown in Fig. 2, Column 3 is assumed to be the arbiter and its decision points to Row 0 in the TM. The scheduler then compares decisions of each column with the Row 0 . Since the results of Column 1 and 2 are included in the base, they are then set to 0 and integrated into the base. Thus, an updated $D M, D M^{*}$, is formed after the sync process. The scheduler will then release the cell with fan-out of 1110 from Input 0 and a copy of the cell in Input 2 with fan-out of 0001 to maximize the output port utilization. These two cells are transmitted to the output through the switch fabric simultaneously. After this time slot of cell transmission, the scheduler will update the $T M$ as described previously. Since there is no iteration in the proposed scheduling algorithm, a complexity of $O(1)$ is achieved, which is suitable for highspeed switches as 100 Gigabit Ethernet switches.

\section{SimUlation RESUltS ANALYSIS}

In this section, we show some simulation results derived from OPNET Modeler [4]. An $8 \times 8$ switch is used to evaluate the performance of the proposed algorithm. Bursty traffic is provided to each input with a mean burst size of 16 cells [5]. Fan-out vectors are uniformly distributed between 2 to 8 with an average fan-out cardinality of 4 , since we do not consider the mixture of unicast and multicast traffic in this study.

Fig. 2 shows the average number of transmissions per cell as a function of traffic arrival rate. Using sync mechanism, the average number of transmissions per cell is significantly reduced and the total number of cells sent to the switch fabric is correspondingly lowered as shown in Fig. 3.

Fig. 4 demonstrates the output port utilization as a function of arrival rate. Nearly the same output port utilization is achieved by both schemes, which shows that the sync does not degrade the utilization to reduce the number of transmissions.

\section{CONCLUSION}

In this paper, we propose a multicast scheduling algorithm for high-speed input-queued switches. Fan-out information of HOL cells is examined by the scheduler and is used to form the Schedule Matrix. Each subscheduler independently executes

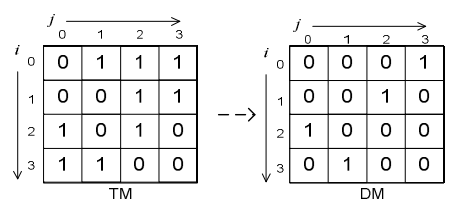

Figure 5. An example of a $4 \times 4$ Traffic Matrix and the Decision Matrix

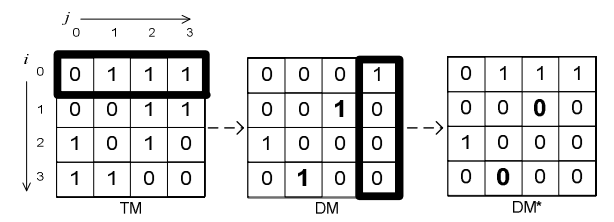

Figure 1. Demonstration of sync process the round-robin algorithm on each column and scheduling decisions result in the Decision Matrix. A sync mechanism is then carried out to reduce the number of transmissions of each multicast cell. Simulation results show that the algorithm effectively performs as designed.

\section{REFERENCES}

[1] Mark J. Karol, Michael G. Hluchyj, and Samuel P. Morgan, "Input versus output queueing on a space-division packet switch", IEEE Transaction on Communications, vol. com-35, no. 12, December 1987

[2] Deng Pan, and Yuanyuan Yang, "FIFO-based multicast scheduling algorithm for virtual output queued packet switches", IEEE Transactions on Computers, vol. 54, no. 10, October 2005

[3] Deng Pan, and Yuanyuan Yang, "Bandwidth guaranteed multicast scheduling for virtual output queued packet switches", Journal of Parallel and Distributed Computing, vol. 69, issue. 12, pp 939-949, August 2009

[4] OPNET Modeler, Available at: http://www.opnet.com/solutions/networr_rd/modeler.html

[5] Balaji Prabhakar, Nick McKeown, and Ritesh Ahuja, "Multicast Scheduling for Input-Queued Switches", IEEE Journal on Selected Area In Communciations, vol. 15, no. 5, June 1997

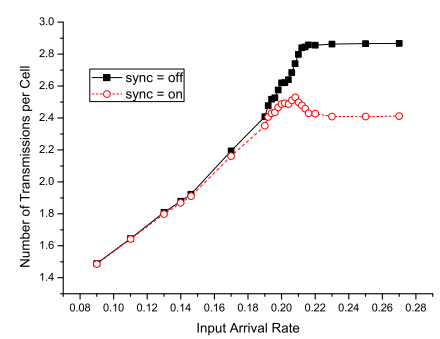

Figure 2. Average number of transmmisions per cell as a function of traffic arrival rate

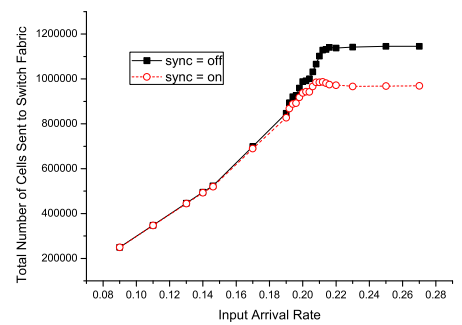

Figure 3. Total number of cells sent to switch fabric as a function of traffic arrival rate

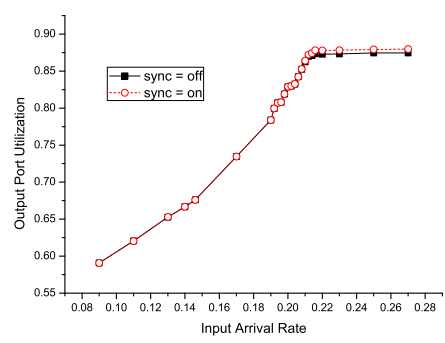

Figure 4. Output port utilization as a function of traffic arrival rate 